\title{
The Nature of the Dissociation Sites of Hydrogen Molecules on Ru(001)
}

\author{
Franck Rose ${ }^{1}$, Mous Tartakhanov ${ }^{1,2}$, Evgeni Fomin ${ }^{1,2}$ and Miquel Salmeron ${ }^{1,3 *}$ \\ ${ }^{1}$ Materials Sciences Division, Lawrence Berkeley National Laboratory, 1 Cyclotron road, Berkeley, CA \\ 94720 USA. \\ ${ }^{2}$ Department of Physics, University of California at Berkeley. \\ ${ }^{3}$ Materials Science and Engineering Department, University of California at Berkeley.
}

* Corresponding author: Prof. Miquel Salmeron, Phone: +1-510-486-6230, FAX: +1-510-486-7268,

Email:mbsalmeron@1bl.gov 


\begin{abstract}
Scanning tunneling microscopy (STM) was used to study the dissociative adsorption of $\mathrm{H}_{2}$ on $\mathrm{Ru}(001)$ near saturation coverage, when the number of residual hydrogen vacancies (i.e., unoccupied $\mathrm{Ru}$ sites) is small. We found that $\mathrm{H}_{2}$ dissociation takes place only on $\mathrm{Ru}$ sites where the metal atom is not bound to any $\mathrm{H}$ atom. Such active sites are formed when at least $3 \mathrm{H}$-vacancies aggregate by thermal diffusion. Sites formed by single $\mathrm{H}$-vacancies or pairs of adjoining vacancies were found to be unreactive toward $\mathrm{H}_{2}$. As a similar phenomenon was found previously on $\mathrm{Pd}(111)$, the present results indicate that the active sites for $\mathrm{H}_{2}$ dissociation share a common characteristic among catalytically active transition metals.
\end{abstract}

\title{
Keywords
}

Active site; Catalysis; Dissociative adsorption; Hydrogen; Ru(001) 


\section{Introduction}

A long-held tenet of heterogeneous catalysis, originating from the Langmuir model of chemisorption [1], is that a surface active site where chemisorption takes place must contain at least as many binding sites as atoms or moieties that are produced when the adsorbed molecules dissociate. This idea is embedded in equilibrium mass balance equations that assume, in the case of the dissociation of a diatomic molecule, that the rate of adsorption is proportional to the square of the fraction of sites that are unoccupied, $(1-x)^{2}$, where $x$ is the surface coverage (with this definition of $x$, the saturation coverage is defined as $x=1$ ) [2]. Another expression of this important idea is the so-called aggregate effect, which refers to the size and structure of the active sites for adsorption or reaction [3-5]. Recently it was shown [6] for $\mathrm{H}_{2}$ adsorbing on $\operatorname{Pd}(111)$, that the metal aggregate formed by pairs of neighboring $\mathrm{H}$-vacancies on a nearly saturated surface, is not reactive toward hydrogen dissociative adsorption. For brevity, we will use the terms "vacancy" or "hydrogen vacancy" to designate a single unoccupied three-fold fcc-hollow Ru site, with no relation to metal atom vacancies. On the $\mathrm{Pd}(111)$ surface, the most active sites for the dissociation reaction are formed when aggregates containing at least three hydrogen vacancies are formed [1, 6-9].

Here, we show that this observation of the peculiar structure of the hydrogen dissociation site is not a unique property of $\operatorname{Pd}(111)$, but that it also applies to another metal such as $\operatorname{Ru}(001)$. Indeed, we found that hydrogen dissociative adsorption on $\mathrm{Ru}(001)$ near saturation coverage follows the very same aggregate effect described by Mitsui et al. [6] in the case of $\operatorname{Pd}(111)$.

Scanning Tunneling Microscopy (STM) was used to unveil the structure of the ordered phases formed by hydrogen on $\mathrm{Ru}(001)$ below $75 \mathrm{~K}$. Our results confirm previous experimental and theoretical descriptions of the system [10-14]. Hydrogen atoms adsorb on three-fold fcc-hollow sites, and ordered phases are observed only below $75 \mathrm{~K}$. Structures with $\sqrt{3} \times \sqrt{ } 3 \mathrm{R} 30^{\circ}, 2 \times 1,2 \times 2-3 \mathrm{H}$ and $1 \times 1$ periodicities are formed with local coverage $\theta$ of $0.33,0.5,0.75$ and 1 monolayer (ML) respectively. The structure of these phases was determined by atomically resolved images [14]. In this paper we present a study of the structure and 
dynamics of vacancy aggregation that produce exposed metal sites where $\mathrm{H}_{2}$ adsorption and dissociation takes place.

\section{Experimental section}

The experiments were carried out with a homemade variable temperature STM [15] cooled by liquid helium in a flow cryostat in an ultrahigh vacuum chamber with base pressure below $5 \times 10^{-10}$ Torr $(1$ Torr $=$ 1.33 mbar). STM images were acquired in the constant current mode with $\mathrm{Pt} / \mathrm{Rh}-30 \%$ tips prepared by electrochemical etching in a molten $1: 4$ solution of $\mathrm{NaCl}$ and $\mathrm{NaNO}_{3}$ at $450^{\circ} \mathrm{C}$. Clean $\mathrm{Ru}(001)$ surfaces ( $2 \mathrm{~mm}$ thick, $10 \mathrm{~mm}$ diameter crystal sample) with large terraces (>100nm in width) were prepared by noble gas ion sputtering and heating cycles between $550 \mathrm{~K}$ and $1100 \mathrm{~K}$. Afterwards, the sample was cooled to $50 \mathrm{~K}$ and briefly flashed to $1100 \mathrm{~K}$ to remove contaminants deposited during the cooling process. STM observations showed that adsorbed impurities and subsurface impurities were present in amounts less than $0.2 \%$ and $1 \%$ respectively. Hydrogen adsorption experiments were realized by introducing hydrogen gas into the chamber to pressures in the range of $10^{-9}-10^{-7}$ Torr.

\section{Results and discussion}

\subsection{High coverage hydrogen structures}

Hydrogen molecules adsorb and dissociate readily on the surface of $\mathrm{Ru}(001)$ at $50 \mathrm{~K}$ forming three consecutive ordered phases as a function of increasing coverage with periodicities of $\sqrt{3} \times \sqrt{ } 3 \mathrm{R} 30^{\circ}, 2 \times 1$, and $2 \times 2-3 \mathrm{H}$ with local coverage of $\theta=0.33 \mathrm{ML}, \theta=0.5 \mathrm{ML}$, and $\theta=0.75 \mathrm{ML}$ respectively [10-14]. Previous experimental and theoretical studies [10-14] have determined that hydrogen atoms are adsorbed on fcchollow threefold sites of the close packed $\mathrm{Ru}(001)$ surface. Our present and past [14] STM observations 
confirm that fcc-hollow threefold sites are the hydrogen adsorption sites on $\mathrm{Ru}(001)$ for all different coverage.

In the STM images hydrogen atoms appear as depressions (dark spots in color or gray scale representations), whereas vacancies (unoccupied Ru sites) are imaged as protrusions (bright spots). The reason for this is the lowering of the tunneling probability on the sites occupied by H. This has also been observed for $\mathrm{H}$ on $\mathrm{Pd}(111)$ [7] and on $\mathrm{Cu}(100)$ [16]. In Figures 1(a) and (d) we present an STM image and a schematic representation of the $2 \times 2-3 \mathrm{H}$ phase. This structure is formed by an ordered lattice of $\mathrm{H}-$ vacancies with $2 \times 2$ periodicity. The bright spots due to the vacancies are separated by sites occupied by hydrogen atoms. Upon completion of the $2 \times 2-3 \mathrm{H}$ phase at $\theta=0.75 \mathrm{ML}$, the uptake rate of hydrogen decreases substantially. To increase the rate we increased the temperature to $75 \mathrm{~K}$ and the $\mathrm{H}_{2}$ pressure to $10^{-7}$ Torr. Under these conditions a $1 \times 1$ hydrogen phase was formed with local coverage $\theta=1 \mathrm{ML}$ (Figures $1 \mathrm{~b}, \mathrm{c}$ and e) amid isolated vacancies and residual patches of the $2 \times 2-3 \mathrm{H}$ phase. Due to the small corrugation of the $1 \times 1 \mathrm{H}$-covered regions $(7 \pm 1 \mathrm{pm})$, compared to the much higher contrast of the vacancies $(35 \pm 2.5 \mathrm{pm})$, it is sometimes difficult to resolve the individual $\mathrm{H}$ atoms. In Figure $1 \mathrm{c}$, the $1 \times 1$ structure of the $\mathrm{H}$ atoms, although with weak contrast, is visible.

\subsection{Diffusion and aggregation of $H$-vacancies}

As mentioned above, to increase the coverage of $\mathrm{H}$ beyond $0.75 \mathrm{ML}$ the sample was heated to $75 \mathrm{~K}$. At this temperature the adsorbate diffusion rate increased so that changes in the images could be observed in the experimental time scale ( 1 min $20 \mathrm{~s}$ image acquisition times). For example, it is easy to locate $\mathrm{H}-$ vacancies that have changed position between consecutive scans as in Figures $1 \mathrm{~b}$ and $1 \mathrm{c}$ (this last image was obtained by scanning a second time the area delimited by the square in Figure 1b). Most importantly, we discovered the formation of aggregates of nearest neighbor vacancies that move collectively during diffusion. This is illustrated in the series of consecutive STM topographs of Figure 2. The vacancy 
aggregates form the fuzzy or streaked triangular regions outlined with dashed lines in the figure. The fuzziness is due to the rapid diffusion of $\mathrm{H}$ atoms and vacancies inside the triangle, as explained in more detail below. The same triangular aggregates were observed by Mitui et al. [6] on the hydrogenated $\operatorname{Pd}(111)$ surface. Some impurities and defects are also present, producing very bright spots (brighter than the hydrogen vacancies) in these images. These impurities, which are stable with time, can be used as reference points and visual guides to follow the changes in the images. The images are snapshots of the process of formation and disintegration (by separation of its components) of vacancy aggregates. In the first and last images (Figures $2 \mathrm{a}$ and d) only $2 \mathrm{~V}$ aggregates ( $n \mathrm{~V}$ designates an aggregate of $n$ vacancies) are observed, while in images $2 \mathrm{~b}$ and $2 \mathrm{c}$ other aggregates such as $3 \mathrm{~V}, 5 \mathrm{~V}, 7 \mathrm{~V}, 8 \mathrm{~V}$, and $15 \mathrm{~V}$ appear.

The structure and the peculiar appearance of the vacancy aggregates in the STM images can be explained with the help of the schematic representations of Figure 2e. When two vacancies come together to form a dimer, they destabilize the $\mathrm{H}$ atom in the nearest adjacent fcc site. At the temperature of the experiment this atom (grey shaded) will jump very easily to one of the vacancies following the most favorable pathway for diffusion, along the fcc-bridge-hcp-bridge-fcc line. The other hydrogen atoms (dark in the schematic) have to go over the top sites or very near another hydrogen atom to access the empty fcchollow site, which is a much less favorable pathway. The higher barrier for this diffusion pathway controls the slow diffusion of the isolated vacancies.

The rapid exchange between the $\mathrm{H}$ atom and the vacancy pair, faster than the imaging time, gives rise to a fuzzy looking triangle enclosing the $3 \mathrm{fcc}$ sites. The argument can be easily extended to larger $n \mathrm{~V}$ aggregates, which appear as a fuzzy equilateral triangle $\mathrm{n}$ atoms by side. Each triangle encloses $n$ vacancies and $n(n-1) / 2 \mathrm{H}$ atoms. An interesting consequence of this diffusive exchange is that triangular aggregates with a 3-fold hollow site at the center, instead of a Ru atom, cannot be formed and have indeed never been observed in either $\mathrm{Ru}(001)$ or $\mathrm{Pd}(111)$ [6-9] (such triangles would point in the opposite direction to the triangles in Figure 2). 
The formation and separation of vacancy aggregates is shown with higher resolution in the images of Figures 3, 4, S1 and S2. Formation of 2V and 3V aggregates is illustrated in Figures 3 and 4 respectively. Aggregates of 4V, 5V, and 6V are illustrated with Figures S1 and S2 in the Supporting Information.

\subsection{Dissociative adsorption of $\mathrm{H}_{2}$}

A major result of the present study is the finding that only Ru metal atoms that do not form bonds with any adsorbed $\mathrm{H}$ atom are active sites for the dissociation of hydrogen molecules (a similar result was reported by Mitsui et al. on $\operatorname{Pd}(111)$ [6]). In the nearly saturated surface these sites can be formed by aggregation of at least three vacancies. In the $2 \times 2-3 \mathrm{H}$ phase (Figure 1) although there are empty sites exposing ruthenium atoms, none of these atoms are contiguous, explaining the drop in dissociative adsorption probability at $\theta=0.75 \mathrm{ML}$ at low temperatures. In order for the catalytic dissociation reaction to continue and the $\mathrm{H}$ coverage to increase, active sites need to be created. This is realized by heating the crystal to activate the diffusion and aggregation of H-vacancies. This phenomenon is illustrated in Figures $3,4, \mathrm{~S} 1$, and S2. In these series of consecutive STM images we show the formation of aggregates of vacancies and their subsequent separation (Figure 3), or their annihilation due to the dissociative adsorption of a hydrogen molecule (Figures 4, S1, and S2). Thanks to the formation of vacancy aggregates, contiguous non $\mathrm{H}$-bonded $\mathrm{Ru}$ atoms are exposed and the $\mathrm{H}$ coverage can increase beyond $0.75 \mathrm{ML}$ so that the $1 \times 1$ hydrogen phase can be formed.

Once formed, the vacancy aggregates decay with a characteristic lifetime. The decay is either by disintegration, i.e., by separation of the constituent vacancies, or by the dissociation adsorption of a $\mathrm{H}_{2}$ molecule annihilating two of the vacancies. This second mechanism is only possible in the presence of gas phase $\mathrm{H}_{2}$, while disintegration can occur both with and without gas phase $\mathrm{H}_{2}$. The three vacancies labeled 1, 2 and 3 in Figure 4a, acquired under $10^{-7}$ Torr of $\mathrm{H}_{2}$, aggregate forming the $3 \mathrm{~V}$ labeled triangle in Figure $4 b$ (the four other vacancies labeled A to D are spectators present before and after the reaction). 
In Figure 4c, the triangular structure has disappeared and is replaced by one remaining vacancy. Since this happened only in the presence of $\mathrm{H}_{2}$ gas, we must conclude that the two missing vacancies have been filled by $\mathrm{H}$ atoms from the dissociation of a hydrogen molecule. Additional examples of formation of triangular-shaped features by vacancy diffusion and their annihilation by hydrogen dissociative adsorption are illustrated with Figures $\mathrm{S} 1$ and $\mathrm{S} 2$ for the case of $4 \mathrm{~V}, 5 \mathrm{~V}$, and $6 \mathrm{~V}$ aggregates. As shown in Figure 5a, we have observed hydrogen dissociative adsorption events in many different types of $n \mathrm{~V}$ aggregates, with $n$ ranging from 3 to 16 . However, and very importantly, a reaction pathway where a $2 \mathrm{~V}$ aggregate is annihilated due to $\mathrm{H}_{2}$ dissociative adsorption has never been observed, exactly as in the case of $\operatorname{Pd}(111)[6]$.

The reason why aggregates of three vacancies (and more) are active toward $\mathrm{H}_{2}$ dissociative adsorption has been explained by theoretical calculations [8] in the case of $\operatorname{Pd}(111)$. The triangular regions generated by $n \mathrm{~V}$ aggregates with $n \geq 3$, can contain metal atoms not bound to any $\mathrm{H}$ atom (Figure 2e). They are generated when three vacancies cluster around a metal atom. In the case of Pd, the DFT calculations indicated that the binding of the metal to $\mathrm{H}$ increases the activation energy of dissociation in the precursor state. In other words, metal atoms bound to $\mathrm{H}$ become "poisoned" $[3,8]$. Three vacancies is the minimal size of vacancy aggregate where a metal atom can be exposed with no direct bonds to any other $\mathrm{H}$ atom. The present results demonstrate that the same mechanism operates in the case of $\mathrm{Ru}(001)$.

Statistics of the vacancy aggregate sizes and reaction pathways are summarized in Table 1 for $2 \mathrm{~V}$ to $7 \mathrm{~V}$ aggregates. Following the same analysis procedure used by Mitsui et al. [6] for $\mathrm{Pd}(111)$, we can compare the data on both metal surfaces. For each $n \mathrm{~V}$ column in Table 1, "number observed" is the total number of individual $n V$ aggregates counted in our STM data. Each of these individual $n \mathrm{~V}$ aggregates correspond to a series of $n_{S T M}$ consecutive images ( 1 min $20 \mathrm{~s}$ image acquisition times per image). "Total image frames" is equal to the total number of images. Each series of $n_{S T M}$ consecutive images has been analyzed to find out the reaction pathway of each individual $n \mathrm{~V}$ cluster, i.e., cluster disintegration or annihilation by dissociative adsorption of a $\mathrm{H}_{2}$ molecule. The total number of events for each reaction pathway appears in 
the rows "Cluster disintegration events" and " $\mathrm{H}_{2}$ adsorption events" of Table 1. For each individual aggregate the disintegration or the annihilation time is measured. Then mean disintegration and annihilation times are calculated and reported in each $n \mathrm{~V}$ column of Table $1 . \mathrm{H}_{2}$ sticking coefficients, $\eta$, were calculated with the formula $\eta=\left(N / N_{0}\right)(2 \pi m k T)^{1 / 2} /\left(P t S_{0}\right)$, where $N / N_{0}$ is the ratio of reacted sites over non reacted sites, $m$ the $\mathrm{H}_{2}$ mass, $k$ the Boltzmann's constant, $T$ the temperature, $P$ the pressure during exposure, $t$ the time of exposure, and $S_{0}$ the surface area of the $n \mathrm{~V}$ site. The number of observed events for the two reaction pathways (disintegration and annihilation) is plotted in Figure $5 \mathrm{a}$ as a function of $n$, or cluster size. In contrast with the case of $\operatorname{Pd}(111)$, where annihilation is preferred over disintegration [6], the two reaction pathways seem to be followed with equal probability on $\mathrm{Ru}(001)$. This branching ratio between reaction pathways is governed by the competition between vacancy diffusion and hydrogen adsorption on the surface. These two processes are dependent on the temperature and $\mathrm{H}_{2}$ pressure. In the case of $\mathrm{Pd}(111)$, the experiments by Mitsui et al. [6] were performed at $65 \mathrm{~K}$ under a $\mathrm{H}_{2}$ pressure of $2 \times 10^{-}$ ${ }^{7}$ Torr. They measured that cluster mean disintegration times were more than two times longer than mean adsorption times in the case of $3 \mathrm{~V}, 4 \mathrm{~V}$, and $5 \mathrm{~V}$ (respectively $710 \mathrm{~s}, 670 \mathrm{~s}, 820 \mathrm{~s}$ and $330 \mathrm{~s}, 150 \mathrm{~s}, 102 \mathrm{~s}$ ). Our experiments on $\mathrm{Ru}(001)$ were carried out at a higher temperature $(75 \mathrm{~K})$, and at lower $\mathrm{H}_{2}$ pressure $\left(7 \times 10^{-9}\right.$ to $1 \times 10^{-7}$ Torr). Consequently, we found that mean disintegration and annihilation times are of the same order of magnitude and almost constant (within the experimental error) for all cluster sizes (Table 1). Time distributions are displayed in Figures $5 \mathrm{~b}$ and $\mathrm{c}$ for the $2 \mathrm{~V}$ and $3 \mathrm{~V}$ cases. The time bin in the graphs corresponds to the time for one STM scan (1 min $20 \mathrm{~s}$ ). As can be seen $90 \pm 5 \%$ of the events occur within the first four minutes of the experiments (first three consecutive STM scans) in our experimental conditions. 


\section{Conclusion}

The dissociative adsorption of $\mathrm{H}_{2}$ on $\mathrm{Ru}(001)$ was studied with STM. Hydrogen adsorbs readily on the surface until the coverage $\theta$ reaches $0.75 \mathrm{ML}$ where an ordered $2 \times 2-3 \mathrm{H}$ structure is formed. The growth rate decreases substantially when the temperature is below $75 \mathrm{~K}$, but increasing the temperature above 75 $\mathrm{K}$ allows the formation of a $\theta=1 \mathrm{ML}$ phase with $1 \times 1$ periodicity. The decrease in growth rate at $0.75 \mathrm{ML}$ is due to the lack of contiguous exposed $\mathrm{Ru}$ atoms. At $75 \mathrm{~K}$ the diffusion rate of $\mathrm{H}$ is high enough to be observable and the dynamics can be followed by STM. We found that aggregates of vacancies facilitate the rapid diffusion of adjacent $\mathrm{H}$ atoms, generating a fuzzy triangular pattern where vacancies and atoms exchange positions rapidly and can generate the sites that are active for $\mathrm{H}_{2}$ dissociation. Only aggregates containing 3 vacancies and more are active, while 2 vacancy aggregates are not. This allowed us to identify the structure of the catalytically active site as one containing a $\mathrm{Ru}$ atom not bound to $\mathrm{H}$, i.e. surrounded by three equilaterally arranged vacancies. The mechanism of the $\mathrm{H}_{2}$ dissociative reaction on adsorbate crowded surfaces involves the aggregation and the concerted diffusion of vacancies that can generate the active site.

\section{Acknowledgements}

This work was supported by the Director, Office of Energy Research, Office of Basic Energy Sciences, Materials Sciences Division, of the U.S. Department of Energy under Contract No. DE-AC02$05 \mathrm{CH} 11231$.

\section{Supporting Information Statement}

Figures S1 and S2: STM topographs showing the formation of vacancy aggregates where $\mathrm{H}_{2}$ dissociative adsorption takes place. This material is available free of charge via the Internet at http://pubs.acs.org. 


\section{References}

(1) Holloway, S. Surf. Sci. 2003, 540, 1-3.

(2) Conrad, H.; Ertl, G.; Latta, E. E. Surf. Sci. 1974, 41, 435-446.

(3) Taylor, H.S. Proc. Roy. Soc. Lond. 1925, A108, 105-111.

(4) Boudart, M. Am. Scientist, 1969, 57, 97-111.

(5) Sinfelt, J.H. Bimetallic Catalysts: Discoveries, Concepts and Applications, John Wiley and Sons, New York, 1983.

(6) Mitsui, T.; Rose M.K.; Fomin E.; Ogletree D.F.; Salmeron M. Nature 2003, 422, 705-707.

(7) Mitsui, T.; Rose M.K.; Fomin E.; Ogletree D.F.; Salmeron M. Surf. Sci. 2003, 540, 5-11.

(8) Lopez, N.; Łodziana, Z.; Illas, F.; Salmeron M. Phys. Rev. Lett. 2004, 93, 146103-1-4.

(9) Salmeron M. Topics in Catalysis 2005, 36, 55-63.

(10) Xu, L.; Xiao, H.Y.; Zu, X.T. Chem. Phys. 2005, 315 , 155-160.

(11) Kostov, K.L.; Widdra, W.; Menzel, D. Surf. Sci. 2004, 560, 130-144.

(12) Shi, H; Jacobi, K. Surf. Sci. 1994, 313, 289-294.

(13) Sokolowski, M.; Koch, T.; Pfnür, H. Surf. Sci. 1991, 243, 261-272.

(14) Tatarkhanov, M; Rose, F.; Fomin, E.; Ogletree, D. F.; Salmeron, M (in preparation).

(15) Behler, S.; Rose, M.K.; Dunphy, J.C.; Mitsui, T.; Ogletree, D.F.; Salmeron, M.; Chapelier, C. Rev. Sci. Instr. 1997, 68, 2479-2485. 
(16) Lauhon, L.J.; Ho, W. Phys. Rev. Lett. 2000, 85, 4566-4569. 
Table 1. Behavior of the Different Vacancy Aggregates (2V to 7V).

\begin{tabular}{|l|l|l|l|l|l|l|}
\hline Vacancy cluster size & $2 \mathrm{~V}$ & $3 \mathrm{~V}$ & $4 \mathrm{~V}$ & $5 \mathrm{~V}$ & $6 \mathrm{~V}$ & $7 \mathrm{~V}$ \\
\hline Number observed & 72 & 82 & 19 & 24 & 13 & 7 \\
\hline Total image frames & 187 & 155 & 66 & 33 & 40 & 9 \\
\hline $\mathrm{H}_{2}$ adsorption events & 0 & 37 & 6 & 14 & 6 & 3 \\
\hline Mean adsorption time (s) & $>1.5 \times 10^{4}$ & 162 & 130 & 114 & 96 & 106 \\
\hline $\mathrm{H}_{2}$ sticking coefficient & $<10^{-6}$ & 0.0027 & 0.0029 & 0.005 & 0.004 & 0.005 \\
\hline Cluster separation events & 72 & & & & & \\
\hline Mean separation time (s) & 207 & 142 & 106 & 104 & 96 & 100 \\
\hline
\end{tabular}


Figures
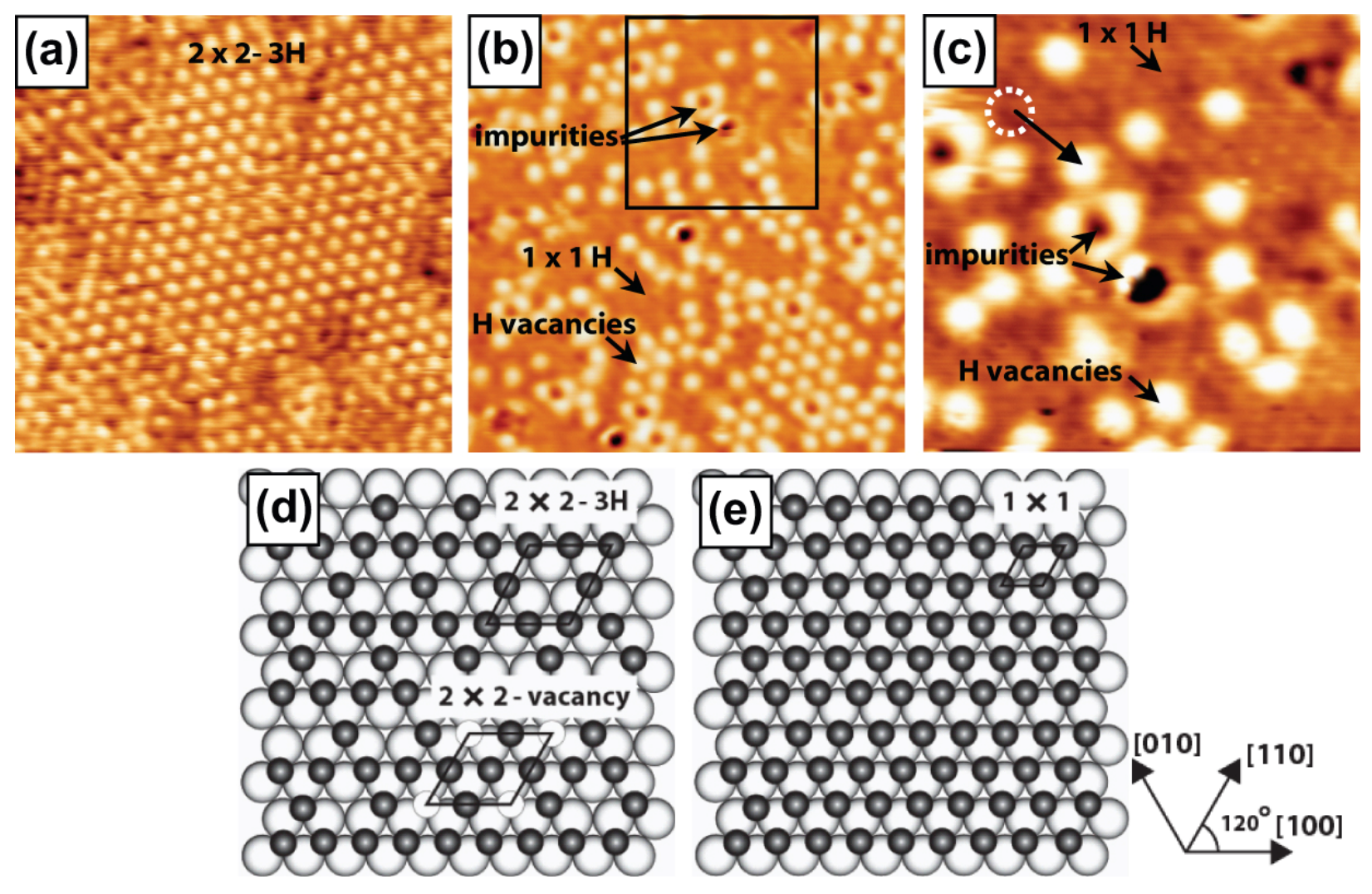

Figure 1 (Color online): STM images of the $\mathrm{Ru}(001)$ covered by $\mathrm{H}$ atoms at a coverage slightly above 75\%. The bright round features correspond to $\mathrm{H}$ vacancies, which appear as $35 \mathrm{pm}$ protrusions. The adsorbed $\mathrm{H}$ atoms have a very low corrugation of approximately $7 \mathrm{pm}$ and are hardly visible. (a) STM topograph of the $2 \times 2-3 \mathrm{H}$ phase $(91 \AA \AA \times 91 \AA$, $25.5 \mathrm{mV}, 6.25 \mathrm{nA})$. (b) STM topograph of a mixed $1 \times 1$ and $2 \times 2-3 \mathrm{H}$ phase $(86 \AA \times 86 \AA, 52 \mathrm{mV}, 1.3 \mathrm{nA})$. A few impurity atoms are also present. (c) Smaller size image acquired $1 \mathrm{~min} 20 \mathrm{~s}$ after (b) in the area marked by the square ( $38 \AA \times 38 \AA, 56 \mathrm{mV}, 1.4 \mathrm{nA})$. The weak corrugation of the $1 \times 1$ hydrogen $(7 \pm 1 \mathrm{pm})$ is visible. One of the several vacancies that have diffused between images (b) and (c) is marked by a circle and an arrow near the top. (d) Schematic representation of the $2 \times 2-3 \mathrm{H}$ phase. (e) Schematic representation of the $1 \times 1$ phase. Large light grey, small black, and small white circles represent $\mathrm{Ru}$ substrate atoms, adsorbed $\mathrm{H}$ atoms, and $\mathrm{H}$ vacancies respectively. 

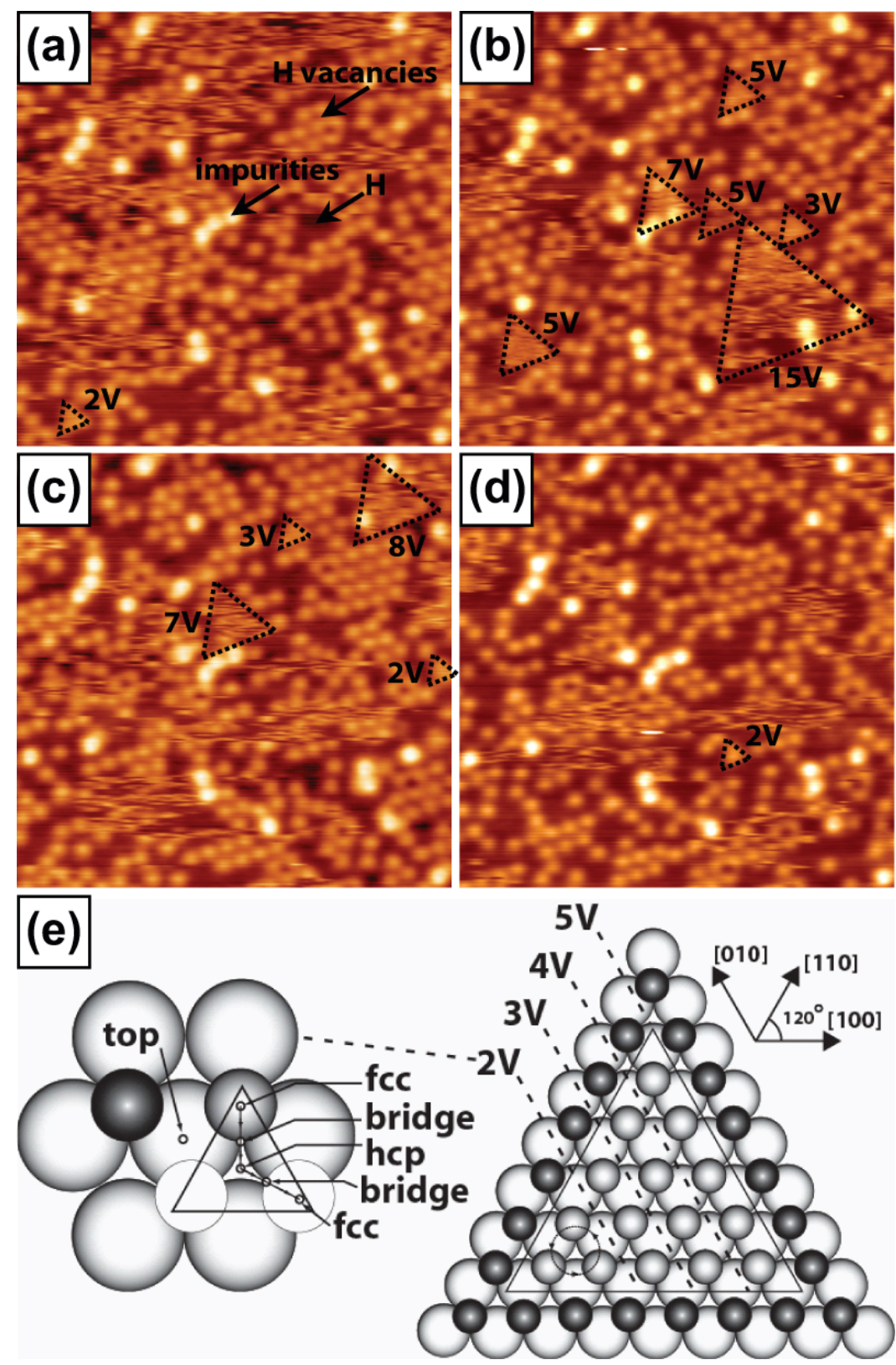

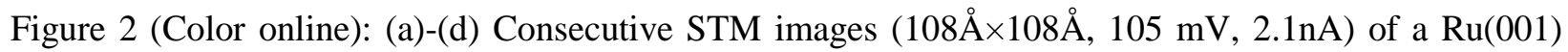
surface with $\mathrm{H}$ at a coverage between 0.76 and 1 monolayer. At the temperature of the experiment, $75 \mathrm{~K}$, the vacancies (bright spots) diffuse and form aggregates containing from 2 to 15 vacancies. Rapid site exchange between adjacent $\mathrm{H}$ atoms and $\mathrm{H}$-vacancies in the aggregate generate streaked or fuzzy looking triangular regions (marked by dotted triangles). A few impurity atoms (bright spots) serve as reference 
points. (e) Schematic representations of the geometry of $\mathrm{H}$ atoms and vacancies: large light grey, small black, and small white circles represent $\mathrm{Ru}$ substrate atoms, adsorbed $\mathrm{H}$ atoms and $\mathrm{H}$ vacancies respectively. $\mathrm{H}$ atoms exchanging positions with vacancies are shown in light gray. The rapid exchange of atoms and vacancies generate equilateral triangular regions $\mathrm{n}$ atoms by side (right hand schematic) containing $n$ vacancies and $n(n-1) / 2 \mathrm{H}$ atoms. Left panel: the most favorable pathway for hydrogen diffusion between neighboring fcc-sites is along the fcc-bridge-hcp-bridge-fcc channel. $\mathrm{H}$ atoms shown in black can only diffuse by hopping over top sites, which has higher activation energy. 
(a)
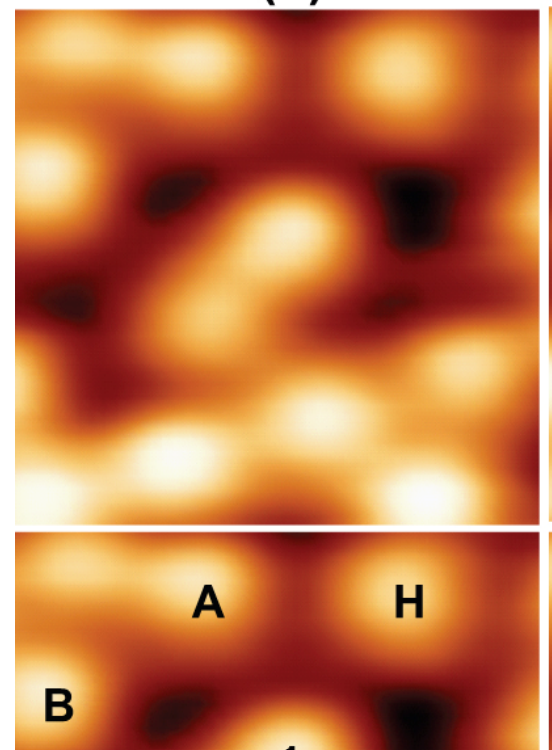

2

C D

E

G

$F$ (b)

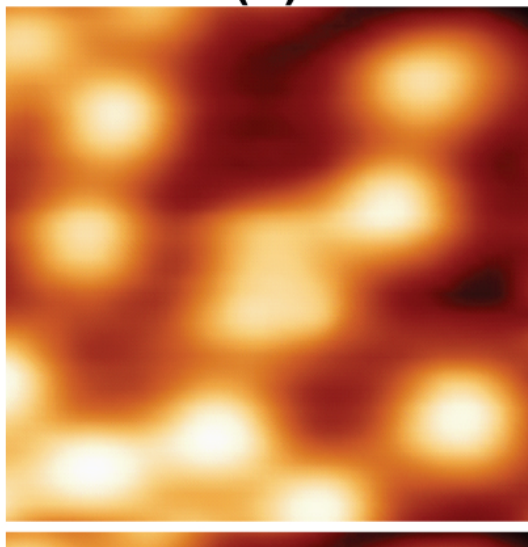

A

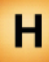

G

B

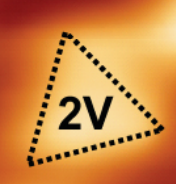

D

C

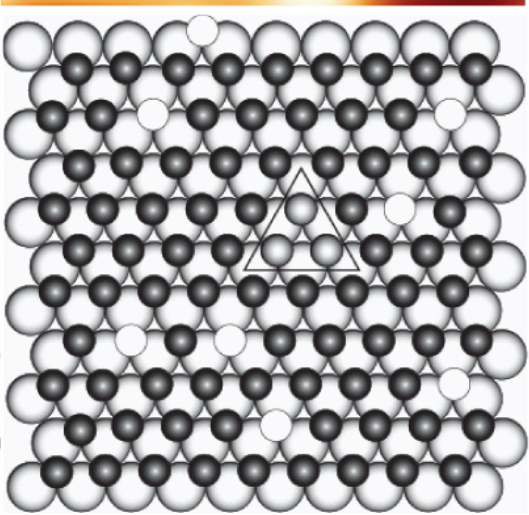

(c)

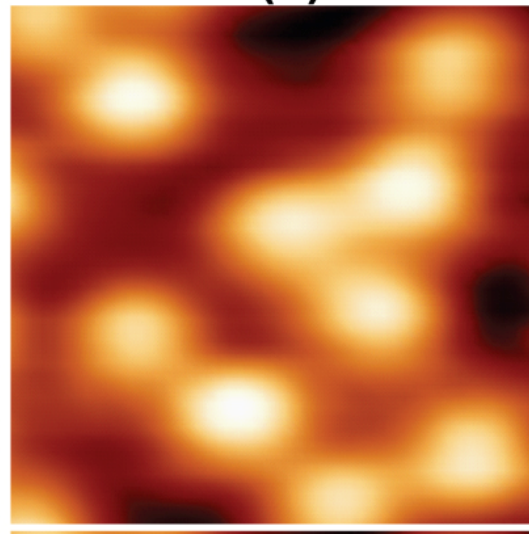

H

A

G
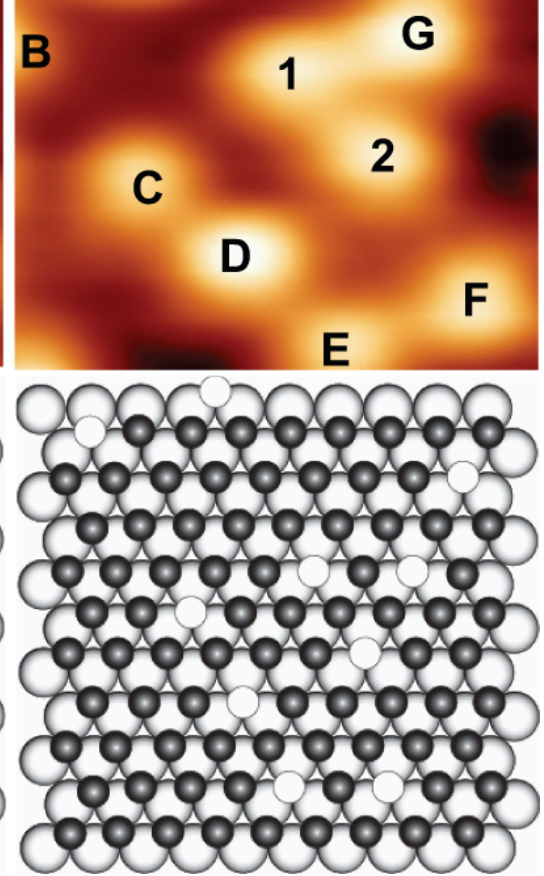

Figure 3 (Color online): STM images showing the formation and separation of $2 \mathrm{~V}$ vacancy aggregates.

The three images are topographs of the same area $(19 \AA \AA \times 19 \AA, 105 \mathrm{mV}, 2.1 \mathrm{nA})$ acquired consecutively.

The same images are shown below with labels to facilitate following the diffusion processes. The bottom row contains schematic representations of each image. (a) Ten vacancies, 1,2,A-H are present. (b) Vacancies 1 and 2 aggregate to form a $2 \mathrm{~V}$ aggregate that is imaged as a triangle due to rapid diffusion. 
Vacancies A to $\mathrm{H}$ remain separated. (c) The $2 \mathrm{~V}$ aggregates separate back into individual vacancies, even in the presence of $\mathrm{H}_{2}$ gas at $10^{-7}$ torr. 
(a)
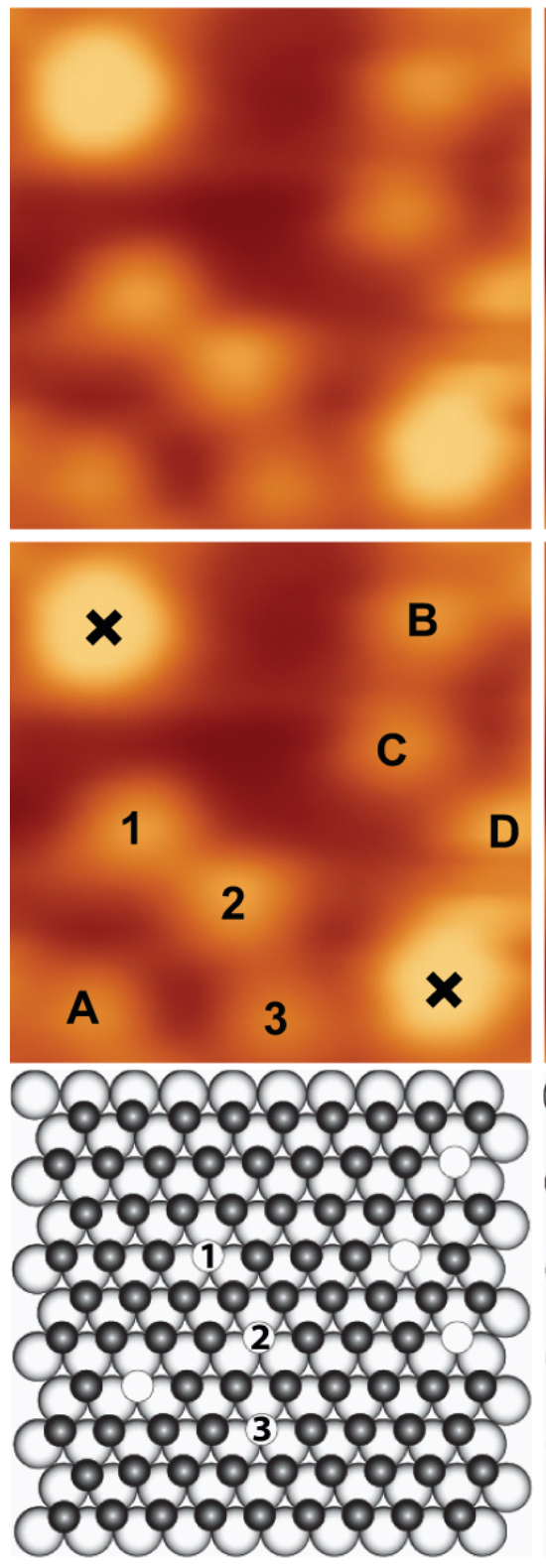

(b)

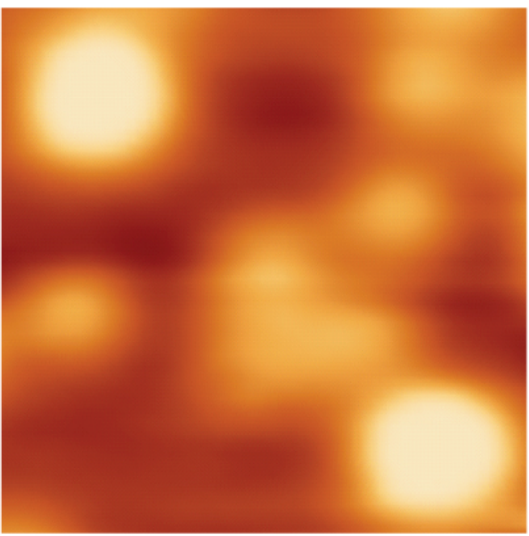

$\times$

A

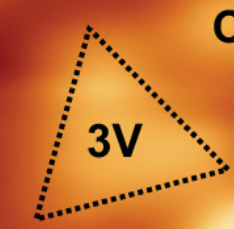

B

C

(c)
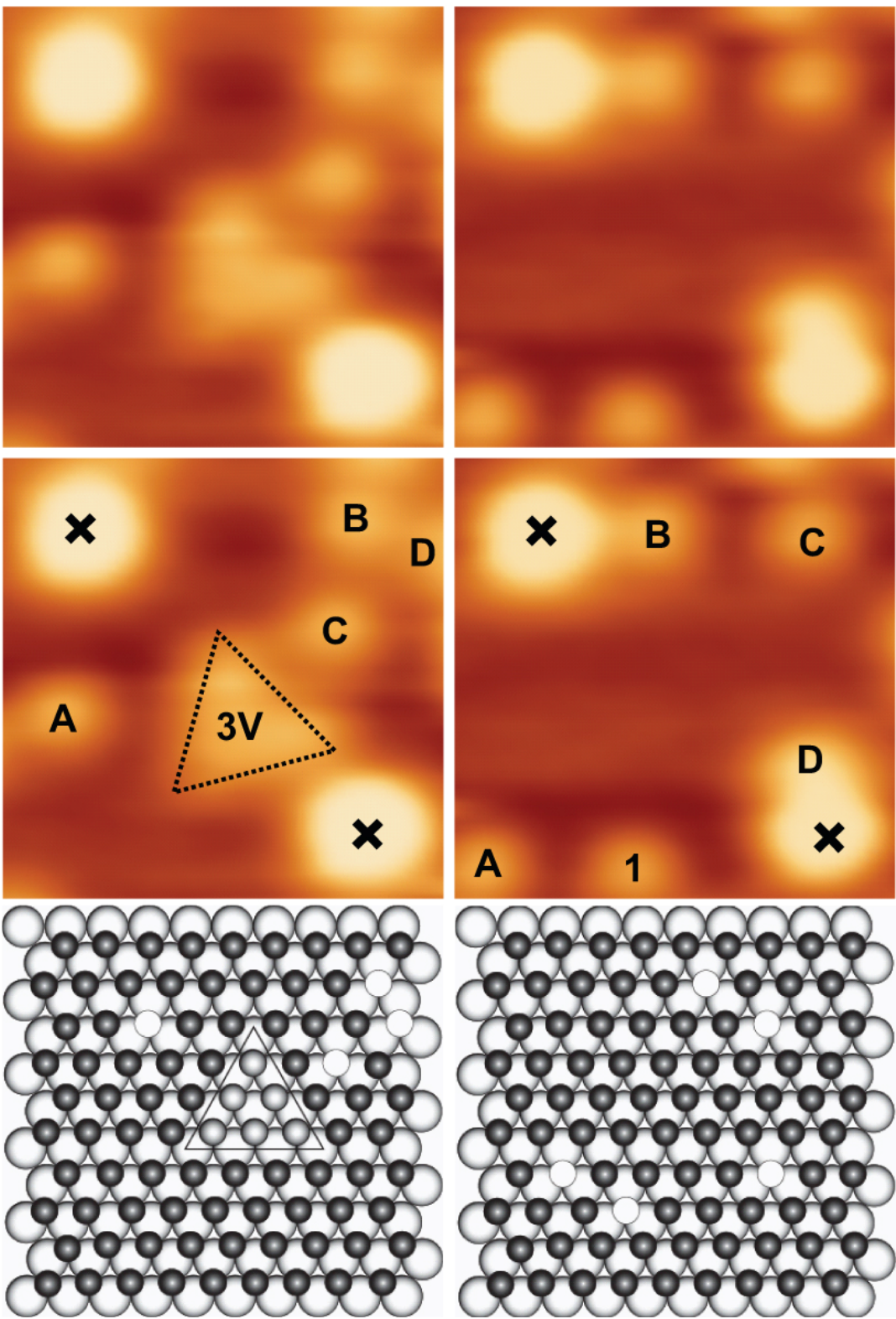

X B

C
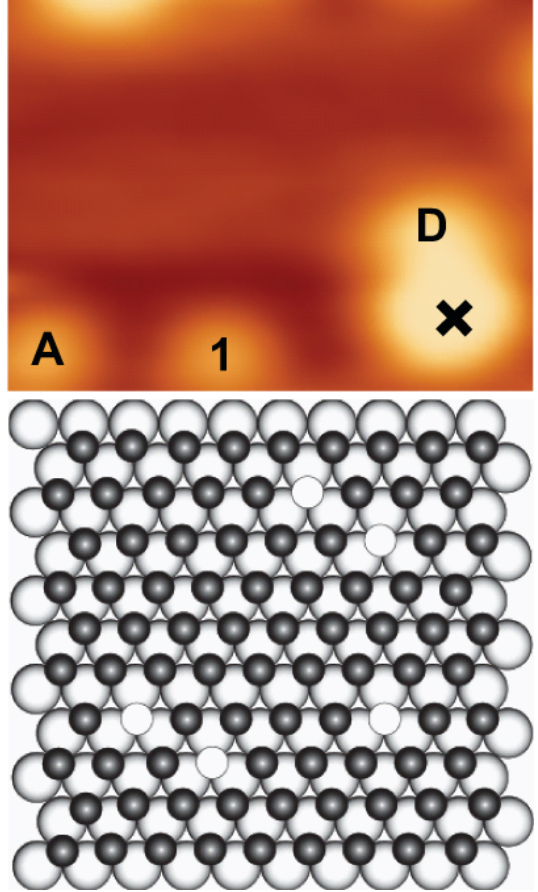

Figure 4 (Color online): Consecutive STM images of the same area (20 $\times 20 \AA, 105 \mathrm{mV}, 2.1 \mathrm{nA})$ showing the aggregation of three vacancies, labeled 1, 2 and 3 in (a) to form a trimer, shown in (b) by a triangle. Annihilation of the trimer occurs by the dissociative adsorption of a $\mathrm{H}_{2}$ molecule from the gas phase leaving one vacancy, labeled 1 in (c). The images are repeated with labels in the second row. Two 
impurity atoms, marked by a cross serve as reference points. The bottom row contains schematic representations of the corresponding atomic structures. Vacancies A to D are spectators. 

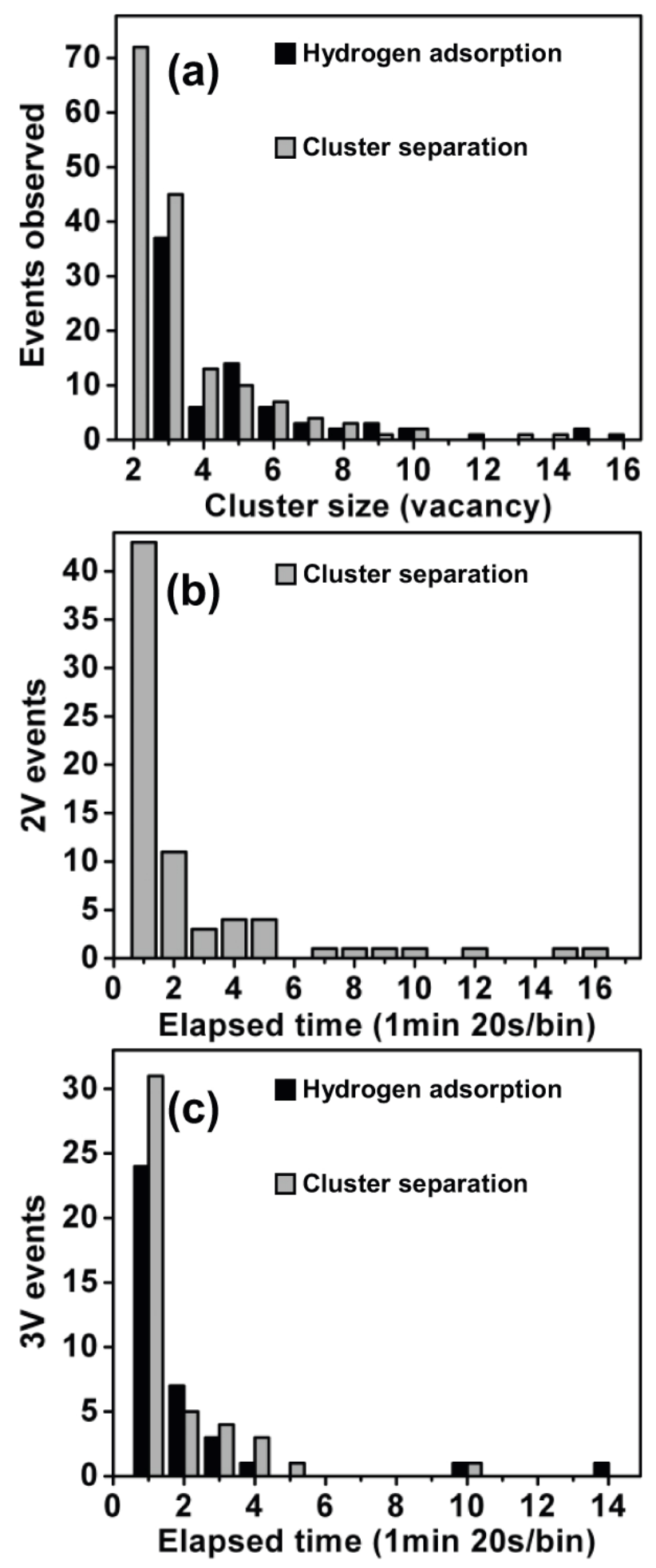
Figure 5: Statistics of observed events for the two possible reaction pathways: cluster disintegration by separation of its components (grey) and annihilation by dissociative adsorption of a $\mathrm{H}_{2}$ molecules (black). (a) Total number of observed reaction events for $n V$ aggregates $(n=2$ to 16). (b) Statistic of the elapsed time for $2 \mathrm{~V}$ cluster separation. (c) Statistic of the elapsed time for $3 \mathrm{~V}$ cluster separation and dissociative adsorption. 
Table of Contents Graphic

$\begin{array}{cccc}\begin{array}{c}\text { monoatomic } \\ \text { vacancies }\end{array} & & \text { B } \\ & & \text { C } & \\ & & & \text { D } \\ & & & \\ \text { A } & 3 & & \end{array}$

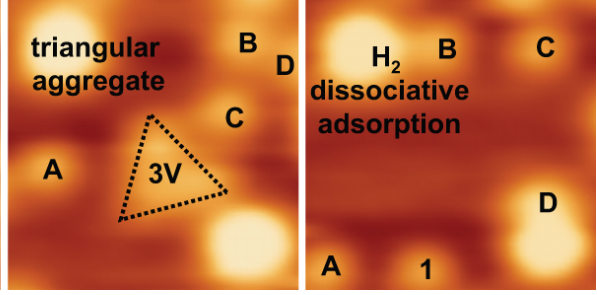

